\title{
Visualisierung versus Sprache
}

\author{
Die Spirale als Metapher oder als Illustration der \\ Phänomenologie des Geistes [1994]
}

In einem Seminar des Winters 1992/93, das die Leistung und Wirkung visueller Darstellungsmittel zum Thema hatte, wurde die Figur der Spirale gleich zu Beginn zum mäeutischen Streitfall. Ich hatte erwähnt, dass in dem neuen dtv-Atlas zur Philosophie Hegels Phänomenologie des Geistes durch eine Spirale veranschaulicht werde, und an dem Vorgehen Zweifel geäußert. In der Frankfurter Allgemeinen sei diese Bebilderung der Philosophie Gegenstand einer heftigen Attacke gewesen.

Eine Studentin widersprach: Das Bild sei angemessen. Hegel selbst verwende das der Leiter. Es sei ihr ganz gegenwärtig und helfe ihr sehr, dieses abstrakte Werk, in das sie sich gerade mit anderen einlese, zu verstehen.

Ein Student ergänzte, durchaus ernst: Wenn solche Bilder Abbreviaturen seien, Konzentrate, könne man da nicht auf den ganzen Wortreichtum der Philosophen, ihr großes Bla Bla verzichten, es streichen? Man hätte dann die Geschichte der Philosophie in einer Figurenreihe. Der Philosoph erschien für einen Augenblick als Magister Ludi, der mit den zu Ideogrammen geronnenen Philosophien wie mit Glasperlen spielt.

Ein zweiter fuhr dazwischen: Die Kritik Gustav Seibts an dem dtv-Atlas, die in der $F A Z$ gestanden habe, sei völlig überzogen gewesen, unsachlich und unfair. Er selbst, wenn er Kant fürs Examen lese, arbeite auch mit Zeichnungen, mit geometrischen Figuren, um ihn sich einzuprägen und die Vielzahl von Gesichtspunkten im Gedächtnis zu behalten. Man müsse bedenken, welches der Zweck des dtv-Atlasses sei: Es sei eine Einführung, die Absicht didaktisch.

Alle drei fühlten sich angegriffen. Eine Frage der Generation? Die Polemik in der FAZ vom 17.1.1992 war überschrieben: „Arbeit am Schwachsinn. - Die neueste Anmaßung der Didaktik: Fastfood - Philosophie in vielen bunten Bildchen." Sie brachte drei Beispiele (siehe im Anhang Abb. 1a-c):

(1) Eine bunte Farbtafel zur Dialektik der Aufklärung von Adorno und Horkheimer. - Odysseus fährt, an den Mast eines Bootes gefesselt, zwischen zwei Felsen hindurch, auf denen Mythos und Aufklärung als Sirenen locken. Zwei Pfeile unter dem Boot in gegenläufiger Richtung deuten an, dass eins in das andere umzuschlagen geneigt ist. Unten links wird ein „Verblendungszusammenhang“ als bebilderter Kreislauf vorgeführt. Die Abstrakta Kulturindustrie, Herrschaft, Warenwirtschaft, instrumentelle Vernunft, wiedergegeben als Donald Duck, Zeigefinger, Geldsack und Computer, kreisen das 
bürgerliche Individuum ein, das rechts noch einmal groß und ziemlich isoliert in der Fläche steht. Rechts unten führt ein kleiner Strich heraus aus dem Gesamtzirkel. „Auswege?“ steht daran.

(2) Eine bräunlich kolorierte Zeichnung zu Nietzsches Kritik der Kultur der Décadence. - Ein tönernes Götzenhaupt, auf dessen Stirn Glaubensgewissheiten wie „freier Wille“, „Christentum“, ,Jenseitsglaube“ geschrieben stehen, wird durch einen Nietzsches Kritik symbolisierenden Blitz in die Mundhöhle zertrümmert, den die Wörter „Täuschung durch Sprache“, „Phantastik“, „Eigennutz"erläutern.

(3) Die graphische Figur zu Hegels Phänomenologie des Geistes. - Eine Spirale, genauer, eine zylindrische Schraube führt in vier Windungen von der Grundstufe „Bewußtsein“ über „Selbstbewußtsein“, „Vernunft“, „Geist“, „Religion“ hinauf zum ,absoluten Wissen“.

Der $d t v$-Atlas zur Philosophie ist eine Geschichte der philosophischen Lehren seit ihren indischen und chinesischen Anfängen und eingeteilt wie die übrigen Bände der seit 20 Jahren bestehenden Reihe: links ein Bildteil, nach Skizzen der philosophischen Herausgeber vom Graphiker des Verlags angefertigt, rechts der Textteil, aus Zitaten und knappen Kommentaren der Editoren zusammengesetzt.

Mit dem Versuch, philosophische Gedanken in Form von Bildern und Graphiken zu veranschaulichen, wird hier weitgehend Neuland für die Darstellung der Philosophie betreten. Die Tafelseiten sollen die Texte anschaulich erläutern, ergänzen oder zusammenfassen. Ihr Ziel ist es, das Verständnis zu fördern und eigenes Fragen anzuregen,

meinen die Verfasser (Burkard, Kunzmann \& Wiedmann 1991: Vorwort). Der Rezensent (Seibt 1992) antwortet, nicht die Kümmerlichkeit des Textteils sei dem Werk anzukreiden: Das Wort bleibe blass und leer und richte nicht viel Schaden an.

Verhängnisvoll ist der Bildteil, denn er verstößt gegen das Bilderverbot, das am Anfang jeder Philosophie steht, so sehr sie sich bildlicher Sprache bedienen muß.

Auf den Einwand, dergleichen sei doch einprägsam, es lasse sich leicht merken, antwortet er:

Genau darin liegt das Verhängnisvolle des neuen Machwerks. Die Abziehbilder dieser Fibel für die Armen im Geiste sind möglicherweise nicht mehr loszuwerden, wenn man sie im falschen, eindrucksfähigen Alter aufgenommen hat. 
Sie schieben sich vor eine Sache und besetzen sie, die als Faktenwissen und Formelsammlung nicht $\mathrm{zu}$ haben ist, sondern allein im Vollzug eines Zusammenhangs und in der Erfahrung einer Anstrengung.

Gustav Seibt zitiert Adornos Theorie der Halbbildung: Das Halbverstandene und Halberfahrene sei

nicht die Vorstufe der Bildung, sondern ihr Todfeind: Bildungselemente, die ins Bewußtsein geraten, ohne in dessen Kontinuität eingeschmolzen zu werden, verwandeln sich in böse Giftstoffe, tendenziell in Aberglauben.

Er schließt:

Der dtv-Atlas zur Philosophie ist nur ein besonders auffallendes Beispiel einer Barbarei, die sich ganz allgemein im Anwachsen der Sphäre des Didaktischen und Aufbereitenden in unserer Bildung anzeigt. Die Ästhetik der Schultafel breitet sich auch sonst in Funkkollegs, Hörsälen und Lehrbüchern aus. Das Element der Geisteswissenschaften aber bleibt die Sprache.

Das Ganze also eine Polemik mit Keulenschlägen; das Seminar hörte sie mit leisem Erschrecken. Sie ließ uns aber eine nicht unwesentliche Frage zurück: Was leistet ein Bild wie die Spirale, um die Phänomenologie des Geistes zu erfassen? Welches sind ihre Eigenschaften? Wo verwendet Hegel das Bild der Leiter, wie gebraucht er es? Welche Gleichnisse verwendet er überhaupt, z. B. in der berühmten „Vorrede“, um die Denkbewegung oder die Grundgedanken seines Werks vorstellbar zu machen?

Allgemeiner gesprochen: Was leisten die Veranschaulichungsmittel, und was bewirken sie? Was haben sie der Wortsprache voraus? Wie weit erschließen sie etwas vom Wort Unerreichbares, und worin bleiben sie dahinter zurück? Was unterscheidet die Visualisierungszeichen von der Sprache der Wörter?

Ich schlug vor, Textpassagen aus der „Vorrede“ und die Spirale aus dem philosophischen Bilderatlas gegenüberzustellen und zu vergleichen. - Was ist eine Spirale?

Der gewöhnliche Sprachgebrauch ist, was die Benennung unsrer Figur angeht, nicht eindeutig. Für den Mathematiker ist sie keine Spirale, sondern eine Schraube.

Bei diesen zylindrischen Schneckenlinien handelt es sich um Kurven, welche weder von einem bestimmten Ursprungsort ausgehen noch ihre Krümmung in ihrem Verlauf verändern. Kletterpflanzen oder Insektentracheen sind dafür charakteristisch,

schreibt Hans Mislin im Katalog zu einer interdisziplinären Schau Die Spirale im menschlichen Leben und in der Natur, die 1985 vom Museum für Gestaltung in 
Basel gezeigt wurde (Hartmann \& Mislin 1985: 11). Ein Freiburger Mathematiker, Klaus Ernhofer, erläutert mir die Entstehung der Schraube aus der Bewegung eines Punktes im Raum. Der Kreis ließe sich mittels zweier Koordinaten beschreiben: Er entsteht aus der Bewegung eines Punktes in einer waagerechten Ebene, die von zwei senkrecht aufeinander stehenden „Impulsen“ bestimmt wird. Die Schraube entsteht, wenn ein dritter, vertikaler Bewegungsimpuls hinzukommt: Der Punkt auf dem Kreis bewegt sich nun in die dritte Dimension auf der Mantelfläche eines Zylinders. So lässt sich jene Figur darstellen, die wir als Schraube oder Sprungfeder kennen.

Mislin (Hartmann \& Mislin 1985: 10f.) unterscheidet von ihr die „echte Spirale“, die er umschreibt:

Unter einer Spiralkurve verstehen wir eine Schneckenlinie, welche von einem bestimmten Punkt ausgeht und während ihrer zunehmenden Entfernung von ihrem Ausgangspunkt ständig an Krümmung abnimmt.

Gemeint ist hier jene flächige Schneckenlinie, die von Archimedes (1972: 1) am Beginn seiner Abhandlung Über Spiralen exakt definiert worden ist und seinen Namen trägt:

Wenn ein Halbstrahl sich innerhalb einer Ebene um seinen Endpunkt mit gleichförmiger Geschwindigkeit dreht, bis er wieder in seine Ausgangsstellung zurückkehrt, gleichzeitig aber sich ein Punkt auf diesem Halbstrahl mit konstanter Geschwindigkeit vom Anfangspunkt des Halbstrahls aus bewegt, so wird dieser Punkt eine Spirale beschreiben.

Stellen wir uns nun vor, dass der Punkt auf dem Halbstrahl sich in vertikaler Neigung zur Ebene vom Anfangspunkt entfernt, dann erhalten wir eine räumliche Spirale entlang der Oberfläche eines Kegels, eines Spitzkelchs. Schraube und kelchförmige Spirale sind die beiden uns hier besonders interessierenden Figuren.

Die mathematische Beschreibung legt nahe, diese räumliche Spirale für besonders geeignet $\mathrm{zu}$ halten, einen historischen Prozess anschaulich $\mathrm{zu}$ machen. Sie verbindet zwei antagonistische Grundbegriffe geschichtlichen Denkens,

- den der zyklischen Wiederkehr, der Kreisläufe, und

- den des Fortschritts, des vertikalen Aufstiegs.

Die Paradoxie der Figur ist früh ausgesprochen worden. „Der Schraube Weg, gerade ist er und krumm“, sagt Heraklit (vgl. Hartmann \& Mislin 1985: 81; Demandt 1978: 242).

Was leistet das Modell, um Hegels Phänomenologie des Geistes anschaulich zu machen? Wenn wir oben sagten, als Kommentar zu der Bildtafel, eine zylindrische Spirale führe von der Grundstufe des Bewusstseins in vier Windungen 
über „Selbstbewußtsein“, „Vernunft“, „Geist“, „Religion“ hinauf zum „,absoluten Wissen“, dann haben wir zunächst nur die Inhaltsangabe des Werks, das aus diesen sechs Kapiteln besteht, mit dem Bild der Spirale verbunden. Aber damit ist die Aussagekraft des Modells nicht erschöpft. Die Figur ist von komplexer Bauart, eine Raumform von vielsinniger und paradoxaler Struktur. Wenn wir nicht Hegels Text, sondern nur die ihm im $d t v$-Atlas beigegebene Illustration zum Ausgangspunkt wählen und zu interpretieren versuchen, könnten wir z. B. sagen:

(1) Die Figur vermittelt das Bild unaufhörlicher, gleichmäßig fließender Bewegung. Die Bewegung verläuft nach einem Muster, einer Formel.

(2) Die Bewegung verläuft in einer aufsteigenden Kreisbewegung, als spiraliger Stufengang.

(3) Die zweite Windung wiederholt analog die erste auf einer höheren Ebene, die dritte die zweite und die erste, die vierte die dritte, zweite und erste.

(4) „Das absolute Wissen“ ist in die Mitte über die vier Windungen gesetzt als schaue es hindurch bis auf den Grund. Anfangspunkt und Endpunkt stehen sich symmetrisch gegenüber.

Wäre das eine zutreffende Beschreibung des Inhalts und der Denkbewegung der Phänomenologie des Geistes? Es gibt bemerkenswerte Parallelen:

(1') In jedem Satz der Phänomenologie drängt sich der Eindruck einer ruhelosen Dialektik des Geistes auf, aber keineswegs nur des gleichmäßigen Fließens. „Zwar ist er nie in Ruhe, sondern in immer fortschreitender Bewegung begriffen. Aber [...]“ (Hegel 1970: 18). - Auf das „Aber“ kommen wir zurück.

(2') Wir finden das Bild des Kreises; es ist, wie schon das Werkregister ausweist, überhaupt nicht selten bei Hegel. Das Wahre „ist das Werden seiner selbst, der Kreis, der sein Ende als seinen Zweck voraussetzt und zum Anfange hat und nur durch die Ausführung und sein Ende wirklich ist“" (Hegel 1970: 23). Ebenso begegnen wir der Vorstellung von der Entwicklung als eines Stufengangs.

Wenn also dieser Geist seine Bildung, von sich nur auszugehen scheinend, wieder von vorn anfängt, so ist es zugleich auf einer höheren Stufe, daß er anfängt. Das Geisterreich, das auf diese Weise sich in dem Dasein gebildet, macht eine Aufeinanderfolge aus, worin einer den andern ablöste, und jeder das Reich der Welt von dem vorhergehenden übernahm (Hegel 1970: 591).

(3') Ein weiteres Moment ist ausgedrückt in dem schönen Wort, das der Basler Mathematiker Jakob Bernoulli (1655-1705), Entdecker der bedeutendsten Eigenschaften der logarithmischen Spirale (,spira mirabilis'), sich als Grabspruch wünschte und das am Fuß seines Epitaphs einer archimedischen Spirale eingeschrieben ist: „EADEM MUTATA RESURGO“ (Fellmann 1985: 22ff.) - dieselbe und verwandelt kehre ich wieder. Es ist heute an einem Pfeiler im Kreuzgang des Basler Münsters zu sehen. Das Motiv des Versinkens in Nacht und der Auferste- 
hung bestimmt die ,Erfahrung des Bewußtseins‘ in seiner Fortbewegung von Stufe zu Stufe, die Reihe der Erscheinungsformen eines Geistes, von dem nie ganz klar ist, ob Hegel in der Phänomenologie den individuellen Geist, d.h. seinen, oder den der Welt meint, und ob er von der Bewegungsform des Geistes in der Geschichte oder von ihrer reflektierenden Erfassung spricht. In jedem Fall gilt für die Aufeinanderfolge seiner Gestalten das berühmte Gesetz der ,Aufhebung“ im doppelten Sinn:

In seinem Insichgehen ist er in der Nacht seines Selbstbewußtseins versunken, sein verschwundnes Dasein aber ist in ihr aufbewahrt, und dies aufgehobne Dasein, - das vorige, aber aus dem Wissen neugeborne, - ist das neue Dasein, eine neue Welt und Geistesgestalt (Hegel 1970: 590).

(4') Schließlich: Am Ende des Werks schaut das absolute Wissen auf die Aufeinanderfolge zurück und durch sie hindurch: „Ihr Ziel ist die Offenbarung der Tiefe, und diese ist der absolute Begriff; [...].“ Hier, im letzten Satz der Phänomenologie, taucht erstmals in leicht verdeckter Form das Bild der Spirale auf, an der Stelle der Ankunft des Geistes bei sich selbst nach dem Durchgang durch seine Gestalten:

nur-

aus dem Kelche dieses Geisterreiches

schäumt ihm seine Unendlichkeit.

Schillers frühes Gedicht Die Freundschaft (1781), dessen Schluss Hegel hier umformt, lässt deutlicher die auch mathematisch bestehende Nähe zwischen Kelch und Spirale erkennen, von der schon die Rede war:

Tote Gruppen sind wir, wenn wir hassen, Götter, wenn wir bebend uns umfassen, Lechzen nach dem süßen Fesselzwang. Aufwärts durch die tausendfache Stufen Zahlenloser Geister, die nicht schufen, Waltet göttlich dieser Drang.

Arm in Arme, höher stets und höher, Vom Mogolen bis zum griech'schen Seher, Der sich an den letzten Seraph reiht, Wallen wir einmüt'gen Ringeltanzes, Bis sich dort im Meer des ew'gen Glanzes Sterbend untertauchen Maß und Zeit.

Freundlos war der große Weltenmeister, Fühlte Mangel - darum schuf er Geister, Sel'ge Spiegel seiner Seligkeit! 
Fand das höchste Wesen schon kein gleiches,

Aus dem Kelch des ganzen Seelenreiches

Schäumt ihm - die Unendlichkeit.

Es ist nachdenkenswert, dass in der Phänomenologie, die reich an Bildern ist, dasjenige der Spirale fehlt und nur am Schluss darauf angespielt wird; - vielleicht deshalb, weil es seiner Konzeption am nächsten kommt und gerade darum irreführend wäre?

Nicht zufällig sagt Sokrates im Gespräch mit Hermogenes, als sich etwas Neues ankündigt: „O, Guter, ich erblicke einen ganzen Schwarm Weisheit“ (Platon, Kratylos: 401e). Im unbegangenen geistigen Gelände sind visuelle Vorstellungen der erste Anhaltspunkt, um Orientierung zu finden; wir werfen Bilder voraus, um mit ihrer Hilfe wie auf Leitern den schwankenden Boden zu betreten. Metaphern sind unter solchen Umständen erste Ordnungshypothesen.

Es scheint mir nicht ausgeschlossen, dass Schillers Gedicht Die Freundschaft und darin die geometrische Figur vom stufenweise aufsteigenden Ringeltanz und schäumenden Kelch bei der Konzeption der Phänomenologie des Geistes Pate gestanden hat. Man könnte auf Parallelen verweisen, was hier nur anzudeuten ist. Goethes ,Urphänomene' sind in den Reichen der Natur beweglich pulsierende, sich abwandelnde Grundmuster, an deren Fassung geometrisch mathematisches Denken Anteil hat. Man betrachte die Notizen während der Sizilienreise von 1787, in denen er die gerade gefasste Theorie der Pflanzenmetamorphose auch als anschaubare geometrische Figur festhält. Die Skizze ist Denkkrücke und Anhaltspunkt (siehe im Anhang Abb. 2a-c). - Bekannter ist, dass erst die Idee der Doppel-Helix es Crick und Watson ermöglichte, die Organisationsform des genetischen Codes zu entschlüsseln, jene Figur der DNS, die zunächst als Skizze und fragiles Demonstrationsmodell existierte und heute ästhetisch und elegant, stabil und beinahe handlich als zweifach gebänderte Wendeltreppe dem öffentlichen Bewusstsein gegenwärtig ist (vgl. die Abb. 9, 11 und 12 im vorherigen Beitrag „Blickprägung und Tatsache“).

Eines ist der ein wenig rätselhafte Umstand, dass ästhetische geometrische Figuren sich als weitreichende Entdeckungsinstrumente und stichhaltige Ordnungsformen bewähren können, ein anderes die Frage ihrer didaktischen Brauchbarkeit und populären Verwendung. Wir sehen an Goethes Notizen das Flüchtige des Entwurfs, das Unruhige und noch Fragile, man glaubt, der Entstehung eines neuen Begriffs zuzuschauen. Ähnliches gilt für eine frühe Skizze im Vorfeld der Doppel-Helix, überschrieben „A Hypothetical Scheme“ (siehe Abb. 9 im vorherigen Beitrag). Wir hätten hier, mit Ludwik Fleck gesprochen, die erste Stufe der ,Entstehung einer wissenschaftlichen Tatsache“ vor uns, die „esoterische“ Wissenschaft, wie sie sich zuerst meist in kleinen Zeitschriftenaufsätzen meldet. 
Flecks Transformationsstufen der Wissenschaft lassen sich im Fall der DoppelHelix insgesamt an ihrer Visualisierung durch Watson beobachten, als zweite der Übergang zum festeren, lehrbaren, didaktischen Modell der „Handbuchwissenschaft" (siehe Abb. 11 im vorherigen Beitrag) und schließlich als dritte Stufe die „Populärwissenschaft“ (siehe Abb.12 im vorherigen Beitrag), die Fleck (1980: 149) wie folgt beschreibt:

Charakteristisch für eine populäre Darstellung ist der Wegfall der Einzelheiten und hauptsächlich der streitenden Meinungen, wodurch eine künstliche Vereinfachung erzielt wird. Sodann die künstlerisch angenehme, lebendige, anschauliche Ausführung. Endlich die apodiktische Wertung, das einfache Gutheißen oder Ablehnen gewisser Standpunkte. Vereinfachte, anschauliche und apodiktische Wissenschaft - das sind die wichtigsten Merkmale exoterischen Wissens.

Das Suggestive am Bild der Spirale ist ihre Universalität. Wir begegnen ihr als Bauform der Natur wie als Denkform und Machform des Menschen. Sie kommt auf allen Stufen der Natur vor, in den Spiralnebeln der Astronomen und den Tiefdruckgebieten der Meteorologen, als Struktur des genetischen Codes und bei Einzellern, in Kletterpflanzen und als Drehwuchs der Fichte, man kennt sie als Schnecke und Widderhorn, als Flugspirale der Vögel, als Bauform der Herzmuskulatur und des menschlichen Innenohrs. Dem steht die Beobachtung der Mathematiker gegenüber, dass vielen dieser Spiralen ein einfaches mathematisches Gesetz zugrunde liegt,

daß die Zahl der Knotenpunkte in einem bestimmten Satz von Spiralen dem Gesetz der Fibonacci-Reihe gehorcht, einer relativ einfachen Beziehung, in der das folgende Glied immer aus der Summe der beiden vorhergehenden gebildet wird: also 1, 1, 2, 3, 5, 8, 13, 21, 34, 55, 89 usw. (Cramer 1988: 195).

Hinzu kommt die direkte Verbindung dieser Reihe zur Regel des Goldenen Schnitts. Von hier aus ist es ein kurzer Weg zu Kunst und Kultus, und wir erwähnen auch hier einige Beispiele: das spiralige Labyrinth beim Kultus der Stadtgründung und die spiralige Anlage von Städten, Wendeltreppen und spiralige Minarette, steinerne Mutterschoßspiralen an Tempeleingängen und Schlangenmandalas, die Spiralbewegung in religiösen Tänzen der Hindus und des Islam, die Jacobsleiter in Gestalt einer Spirale (Blake) oder der Aufstieg im Purgatorio nach dem gleichen Muster (Dante), der zwölfjährige Christus, auf dem Gipfel einer Steinserpentine thronend und lehrend. Der Katalog jener oben erwähnten Basler Ausstellung von 1985 und ein Werk von Jill Purce, Die Spirale Symbol der Seelenreise, bieten hier kaum erschöpfliches Material. Es ist die Isomorphie subjektiver und objektiver Sphären, die an dieser Figur so anzieht: als existiere eine prästabilierte Harmonie zwischen Natur und menschlichem Geist. 
Freilich gibt es in den erwähnten Veröffentlichungen auch voreilige Harmonisierungen; noch auffälliger ist, dass die geschichtliche Welt von der Vorstellung einer spiraligen Bauform fast ausgenommen ist. Das gilt nicht für die individuelle Geschichte der Seele, hier ist sie beliebt, allerdings eher als Symbol eines Weges denn als Bild einer einmaligen Entwicklung. Ein Aufsatz aus dem Basler Katalog Die Spirale als Modell der Entwicklung (Hartmann \& Mislin 1985: 69ff.), gemeint ist die Jugendentwicklung, wirkt schwammig; die kühne Ausnahme für die Spirale als Geschichtsbild macht Schelling: „Die Weltenjahre zeigen einen Spiralverlauf, der Weltzyklus läuft in einer sich erweiternden Spirale ab“ (Hartmann \& Mislin 1985: 69; vgl. Demandt 1978: 242, 252f., 258).

Ein älterer Bildtyp, zu finden in anonymen hermetischen Manuskripten des 12. Jahrhunderts (Purce 1988, Abb. 34 u. 36, Bibliothèque Nationale, Paris, und National Gallery of Scotland), verdient besondere Aufmerksamkeit (siehe im Anhang Abb. 3). Die Seele schraubt sich empor zu Gott, der über der Welt thront. Die spiralige Bewegung wird dargestellt durch eine Kette tanzend aufsteigender und einander die Hände reichender Figuren, deren Weg auf der Ebene von Erde und Wasser beginnt, Luft und Feuer, die Sphären der sieben Planeten, der Fixsterne und Himmelsbeweger durchquert, danach auf der weiteren Wanderung die Pflanzen-, Tier-, Vernunft- und Himmelsseele hinter sich lässt, die zehn Stufen der Erkenntniskräfte und der ihnen korrespondierenden Engelschöre, um auf der Ebene nur potentieller Materie und Form vor Gottes Thron $\mathrm{zu}$ gelangen. Die Majestas Domini thront über einem durch den aufsteigenden Ringeltanz gebildeten Kelch. Die Nähe des hermetischen Bildtyps zu Schillers Gedicht Die Freundschaft, das von der Forschung „nur“ mit Spinoza, Leibniz und der Philosophieund Literaturgeschichte des 18. Jahrhunderts in Verbindung gebracht wird (Schiller 1991: 102-106), ist recht frappierend, und auch der Schluss der Phänomenologie des Geistes wirkt, als könnte Hegel nicht nur von Schillers Gedicht angeregt sein (man vergleiche z.B. eine Figur wie den vom Auge Gottes gekrönten „Philosophischen Baum“ des Athanasius Kircher (1981, Abb. 4.2):

Das Ziel, das absolute Wissen oder der sich als Geist wissende Geist hat zu seinem Wege die Erinnerung der Geister, wie sie an ihnen selbst sind und die Organisation ihres Reichs vollbringen. Ihre Aufbewahrung nach der Seite ihres freien in der Form der Zufälligkeit erscheinenden Daseins, ist die Geschichte, nach der Seite ihrer begriffenen Organisation aber die Wissenschaft des erscheinenden Wissens; beide zusammen, die begriffene Geschichte, bilden die Erinnerung und die Schädelstätte des absoluten Geistes, die Wirklichkeit, Wahrheit und Gewißheit seines Throns, ohne den er das leblose Einsame wäre; nur aus dem Kelche dieses Geisterreiches schäumt ihm seine Unendlichkeit. 
Wie wir eingangs sahen, gibt es etliche Eigenschaften der Schraube oder der sich öffnenden Spirale, die sich zu Hegels Werk in Parallele setzen lassen. Die zuletzt besprochene hermetische Bildformel der zu Gottes Thron aufsteigenden Seelenspirale kommt, auf wie vielen Überlieferungswegen auch immer, geradezu als Vorbild des Schlusses der Phänomenologie des Geistes in Frage. Die Figur der Schraube im $d t v$-Atlas, von der wir ausgingen, verhilft zu Einsichten und wäre insofern gut gewählt. Aber gerade das könnte dazu verführen, die Parallelisierung $\mathrm{zu}$ weit $\mathrm{zu}$ treiben, die Illustration für die Sache $\mathrm{zu}$ halten, und hier sperrt sich der Text des Werks und seine Sprache.

Es macht die große Schwierigkeit, vielleicht die Crux der Phänomenologie des Geistes aus, dass sie im gleichen Augenblick die Geschichte und ihre systematische Erfassung zum Thema macht, die geschichtlich zufällige Erfahrung des Bewusstseins, das auszieht, eine Eselin zu suchen, und die hinter seinem Rücken stattfindende Lenkung, die dahin führt, dass es ein Königreich findet, das Unvereinbare: Zufall und Ziel, die blinde Aufeinanderfolge von Geisteserscheinungen und ihre als Verbalsubstantiv, als Handlung und Vorgang aufgefasste ,Organisation', dass also seine Form ein nicht auflösbares Paradox ist: offen und geschlossen zugleich (vgl. Hippolite 1973: 46ff.). Der zitierte Schlusssatz, der genau dies zum Thema macht, entwirft denn auch nicht ein einheitliches zusammenschließendes Denkmodell, sondern zeichnet sich aus durch eine sprunghafte Bilderfolge, an deren Schluss kurz das Bild des Kelches auftaucht.

Diese Ausdrucksweise ist charakteristisch. Die eingangs erwähnte „Leiter“ ist ebenso wenig wie die Spirale ein Denkmodell Hegels, sie wird gelegentlich, durch den Kontext eng und konkret umgrenzt, im Sinn von ,Hilfe‘ gebraucht: Das Individuum habe das Recht zu fordern, „dass die Wissenschaft ihm die Leiter wenigstens zu diesem Standpunkte reiche“ (Hegel 1970: 29). Das auffälligste Stilmittel ist ein manchmal allmählicher und oft sprunghafter Wechsel der Bilder (Hegel 1970: 18f.):

Es ist übrigens nicht schwer zu sehen, daß unsere Zeit eine Zeit der Geburt und des Übergangs zu einer neuen Periode ist. Der Geist hat mit der bisherigen Welt seines Daseins und Vorstellens gebrochen und steht im Begriffe, es in die Vergangenheit hinab zu versenken, und in der Absicht seiner Umgestaltung. Zwar ist er nie in Ruhe, sondern in immer fortschreitender Bewegung begriffen. Aber wie beim Kinde nach langer stiller Ernährung der erste Atemzug jene Allmählichkeit des nur vermehrenden Fortgangs abbricht - ein qualitativer Sprung - und jetzt das Kind geboren ist, so reift der sich bildende Geist langsam und stille der neuen Gestalt entgegen, löst ein Teilchen des Baues seiner vorhergehenden Welt nach dem andern auf, ihr Wanken wird nur durch einzelne Symptome angedeutet; der Leichtsinn wie die Langeweile, die im Bestehenden einreißen, die unbestimmte Ahnung eines Unbekannten sind Vorboten, daß etwas anderes im Anzuge ist. Dies allmähliche Zerbröckeln, das die Physiognomie des Ganzen nicht veränderte, wird durch den Aufgang unterbrochen, der, ein Blitz, in einem Male das Gebilde der neuen Welt hinstellt. 
In den wenigen Sätzen, deren rhythmischer Periodenbau von seltener Schönheit ist, lassen sich etwa zehn Vorstellungsbezirke deutlich unterscheiden, denen Hegel die Bilder entleiht, um mit ihrer Hilfe das Thema des Epochenumbruchs zu beleuchten. Die Bilder zielen auf dasselbe, sind aber kaum miteinander vereinbar, Geburt und Abbruch von Verhältnissen, Sprung, Baufälligkeit und Blitz. Sie sind auch schwerlich kongruent mit dem Bewegungstyp der Spirale, haben keineswegs in diesem Modell einen gemeinsamen Nenner, zielen eher auf abrupte Veränderung: „Aber wie beim Kinde [...] der erste Atemzug [...] ein qualitativer Sprung [...].“

Wir fassen hier, an dieser Stelle, einen tiefgreifenden Unterschied zwischen der Sprache der Wörter und den visuellen Zeichen. Wörter sind nur andeutend. Die Metapher meint in der Regel keine durchgehende Analogie, sie ist, zumindest hier, auch weniger als ein Modell. Es gibt da, mit Karl Bühler gesprochen, ein Prinzip der abstraktiven Relevanz. Bühler wählt das einfache Beispiel des Salonlöwen. In der Metapher werden zwei auseinanderliegende Sphären sprunghaft, kurzschlussartig miteinander verbunden, in der Weise, dass die Sphären, die vereint werden, einander teilweise abdecken und wechselweise eine selektive Wirkung üben. Die Sphäre ,Salon' deckt an der des ,Löwen' seine Blutgier und den Gestank aus seinem Munde ab, am Hölzlekönig, einem hohen Baum des Südschwarzwalds, erwarte ich keinen Purpurmantel, sondern seine überragende Statur. Eine Metapher ist ein Pfeil, der den Gegenstand anzielt, kein ihn erschöpfendes Abbild. Wenn Hegel (1970: 19) schreibt:

Wo wir eine Eiche in der Kraft ihres Stammes und in der Ausbreitung ihrer Äste und in den Massen ihrer Belaubung zu sehen wünschen, sind wir nicht zufrieden, wenn uns an Stelle dieser eine Eichel gezeigt wird,

meint er nicht, dass die Phänomenologie des Geistes nach Analogie einer Eiche aufzufassen sei, sondern etwas viel Abstrakteres: ,Das Wahre ist das Ganze‘. Die Metaphern oder Bilder verlieren durch das Phänomen, auf das sie angewandt werden, an Bildgehalt, der jeweilige Zusammenhang gibt ihnen einen abstrakteren und engeren Sinn. Sie sind ein Erfassungsversuch, nennen einen Aspekt des Gegenstandes, der in eigentlicher Sprache nicht recht zu fassen wäre.

Das Gesetz der Abdeckung funktioniert nach Bühler allgemein, er illustriert es am Beispiel eines Differenzbildes (siehe im Anhang Abb. 4a). Ich meine allerdings nicht, dass dieses Bild des Ineinanderschiebens und Einanderabdeckens zweier Diapositive für die Metapher zutrifft; es ist zu starr, tatsächlich bleibt ein Rest, aus der Reaktion der beiden Vorstellungsbezirke aufeinander resultierend, welcher das Eigentümliche des metaphorischen gegenüber dem bildlosen Begriff ausmacht. Auf diese Weise kann sie zur „schöpferischen Ordnungshypothese“ werden. Wilhelm Köller (1975: 201) hat Bühler (1965: 348f.) einer genauen Kritik unterzogen. 
Und was ergibt sich, wenn der Gegenstand, der durch die Metapher beleuchtet werden soll, unanschaulich ist oder eine unklare Bildwirkung von ihm ausgeht? Wenn also keine Vorstellungsbezirke sich verschränken? Wenn man kaum eine Vorstellung oder gar kein Wissen von dem Bildempfänger hat? Dann kann das Prinzip der wechselseitigen Abdeckung, der Selektion nicht wirksam werden. Das könnte der Fall sein bei der Spirale der Phänomenologie des Geistes, bei aller Philosophie, die etwas so Entzogenes wie das Denken selbst zum Gegenstand macht. Das Bild erhält die Chance, sich ungehemmt durchzusetzen und den Begriffsgehalt zu bestimmen, alles Weiterfragen abzuschließen und als suggestive Scheinlösung zu figurieren. Für das illustrierende Modell gilt das in erhöhtem Grad. Vielleicht steht darum das Bilderverbot am Anfang aller Philosophie?

Hier tritt der Bilderwechsel ein. Das Verfahren ist eine Sonderform der Synonymenvariation, der Einkreisung eines Phänomens durch eine Reihe bedeutungsähnlicher Wörter, wie ich sie am Beispiel von Goethes Schreibweise in der Metamorphose der Pflanzen darzustellen versucht habe (vgl. Pörksen 1986a: 82ff.). Durch die Reihe der Bilder wird eine Anzahl mehr oder weniger ähnlicher Bedeutungsaspekte aktualisiert. Die variierenden Bilder ergänzen und verdeutlichen einander zudem wechselseitig - Hegel nähert sich in dem zitierten Absatz von verschiedenen Seiten dem gemeinten begrifflichen Inhalt. Zusammen entwerfen sie hier den Begriff des Epochenumbruchs. Die Bilder verdeutlichen und ergänzen einander nicht nur, zugleich wird durch das Variieren jedes einzelne relativiert, als nicht endgültiges abgelöst. Dadurch bleibt der Begriffsinhalt zugleich unbestimmt offen. Der Eindruck eines vagen, die Sache offenhaltenden Sprechens entsteht. Die Sprache wird als Annäherungsinstrument sichtbar und relativiert.

Wir können also auch auf den Bilderwechsel, der in vergleichbarer Weise gelegentlich von Goethe, häufiger von Freud praktiziert wird, den Begriff der ,Namenbündelung، anwenden, der bei Peter Hartmann auftaucht (siehe im Anhang Abb. 4b). Auch hier darf man die Abbildung nur in ihrem abstraktesten Sinn nehmen. Die verschiedenen Namen geben dem Gegenstand eine jeweils unterschiedliche Interpretation, sie sind ein Bündel von Nuancennennern. Als Träger einer ,Vorstellung' ist jeder nur ein begrenzter Erfassungsversuch. Wort und Sache werden bei diesem Verfahren der Bildervariation auseinandergehalten, die Kluft zwischen ihnen bleibt bewusst.

Das einzelne Bild, das einzelne durchgehaltene Denkmodell fixiert dagegen den Gegenstand, es verführt dazu, das Darstellungsmittel und den Gegenstand nicht mehr auseinanderzuhalten, Modell und Sachverhalt zu verwechseln. Auch das wiederum gilt sehr viel mehr, wenn das Darstellungsmittel eine Zeichnung, ein sichtbares oder greifbares Modell ist. Der Metaphernreichtum dagegen ermöglicht als offene Form die Offenheit des Inhalts. Diese Form regt den Leser fortwährend an, das nicht ganz, nicht endgültig Ausgesprochene zu ergänzen. Er 
wird am Aufbau des Gegenstandes beteiligt, wird stimuliert, ihn in ungezählten Variationen zu präzisieren, ohne seiner je ganz habhaft zu werden. Bei einem Metapherncolloquium in Oldenburg war für Rudolf zur Lippe dies der entscheidende Aspekt.

Es ist das Gegenteil einer Didaktik, die fertige Resultate zur Verfügung stellt. Als didaktisches Modell zur Illustration der Phänomenologie des Geistes ist die Schraube in der Tat gefährlich. Sie kann den Anschein erwecken, eine komplexe Bauform, einen vieldimensionalen dynamischen Zusammenhang auf einen Blick in einigen Hauptzügen durchschaubar $\mathrm{zu}$ machen, räumlich und dinglich vorstellbar, synoptisch fassbar, dem Gedächtnis griffbereit. Diese Dinglichkeit ist ihre Schwäche. Die sinnliche Objektivierung erweckt unsinnigerweise den Schein größerer Objektivität, von ihr geht eine gewisse Bannkraft aus. Während des erwähnten Colloquiums war es auffällig, wie sehr sich die Diskussion an der in der Mitte des Kreises liegenden Spirale festsaugte. Eine Metapher dagegen führt ihr Anführungszeichen mit sich.

Die Möglichkeit, mit Hilfe des Modells der Spirale zu schnell zu verstehen und gerade dadurch misszuverstehen, liegt auf der Hand. Es vermag der von Hegel intendierten zugleich geschichtlichen und systematischen Wirklichkeit nicht gerecht zu werden. Folgen wir seiner Denkbahn einen Augenblick als einem starren Perspektiv: Die Geschichte des Geistes, des einzelnen oder der Menschheit, wäre dann ein konsequenter Stufengang, gleichförmig fließend und nach einem Muster verlaufend. Bewusstsein - Selbstbewusstsein - Vernunft - Geist - Religion Absolutes Wissen wären auseinander folgende und einander spiegelnde Bildungen. Eine öde Monotonie und tote Symmetrie wäre das Resultat. In der konsequent befolgten geometrischen Figur wird eine Ästhetik und totalitäre Vergewaltigung der Geschichte erkennbar, eine Dogmatik, die der später von Hegel theoretisch ausgearbeiteten „systematischen Weltverfassung“ nicht ganz fernlag und noch weniger einer in seiner Nachfolge ausgearbeiteten Welt.

Die Phänomenologie des Geistes ist durch ein einziges Dingmodell nicht darstellbar. Auch die Spirale würde stilisieren. Daher die Vorzüge der in immer neuen Ansätzen offenen Form, des nur andeutenden Metaphernsprungs. 


\section{Abbildungen}

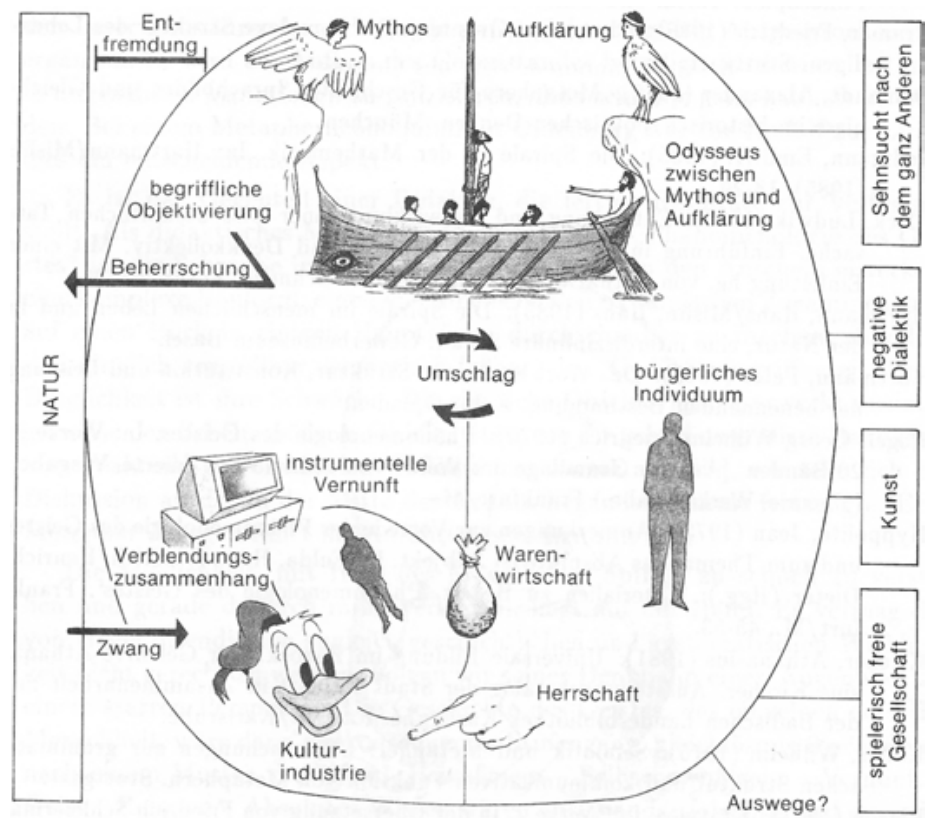

Abb. 1a: Dialektik der Aufklärung (aus: dtv-Atlas zur Philosophie. München 1991: 226).

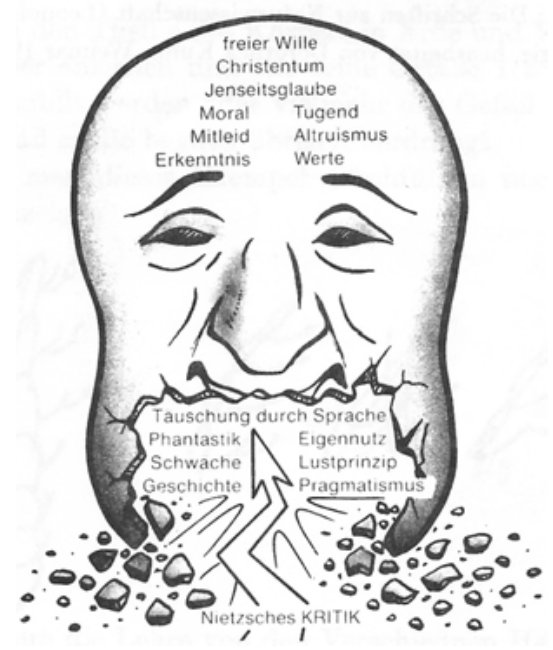

Abb. 1b: Nietzsches Kritik (aus: dtv-Atlas zur Philosophie. München 1991: 176)

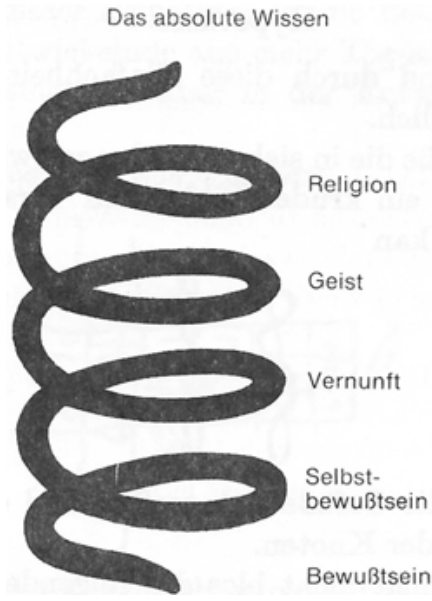

Abb. 1c: Phänomenologie des Geistes (aus: dtv-Atlas zur Philosophie. München 1991: 156) 


\section{Hypothese}

Alles ist Blat, und durch diese Einfachheit wird die gröBte Manigfaltigkeit möglich.

Das Blat hat gefäße die in sich verschlungen wieder ein Blat hervorbringen wo man ein krudes Bild durch Verschlingung zweyer 155 Linien sich formiren kan

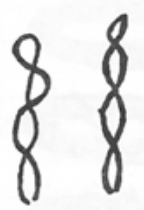

Der Punckt wo die Gefäße sich treffen und dies neue Blat zu bilden anfangen ist der Knoten.

Dieser Knoten bringt nicht blos das folgende Blat hervor sondern mehrere.

Ein Blat das nur Feuchtigkeit unter der Erde einsaugt nennen wir Wurzel, Ein Blat das von der Feuchtigkeit ausgedehnt wird pp. Zwiebel. Bulbus.

Ein Blat das sich gleich ausdehnt einen Stiel. Stengel.

Abb. 2a: Skizze zur Metamorphose (aus: J.W. von Goethe, Die Schriften zur Naturwissenschaft (Leopoldina). Bd. 9A: Zur Morphologie. Bearb. von Dorothea Kuhn. Weimar 1977: 58)

Der Hauptgrund dieser Hypothese ist die Betrachtung daß der 165 Keim oder das zu entwickelnde aus mehr Theilen besteht die mit einander verwandt sind sich aber in der Entwicklung einander aufheben z.E.

Der Körper a b. bestände aus 6 fächern

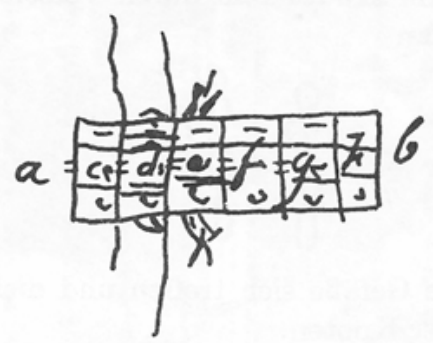

die von Natur einander alle gleich wären gleiches Maßßes gleicher 170 Beschaffenheit jeder dieser Theile hat zwey seiten nach außen außer c. und $h$ welche außerdem noch eine Seite nach a und b. haben.

Abb. 2b: Skizze zur Metamorphose (aus: J.W. von Goethe, Die Schriften zur Naturwissenschaft (Leopoldina). Bd. 9A: Zur Morphologie. Bearb. von Dorothea Kuhn. Weimar 1977: 58f.) 
Wir setzen den Theil c des Körpers in Erde und Wasser so wird 175 er nun Wasser anziehen und alle seine Gefäße 123 werden mit Wasser ausgefüllt werden oder vielmehr das Gefä $\beta 1$ wird sich so ausdehnen daß es die beyden übrigen verdrängt.

Nun muß man dieses Exempel durchführen noch besser aber dergestalt anzeigen
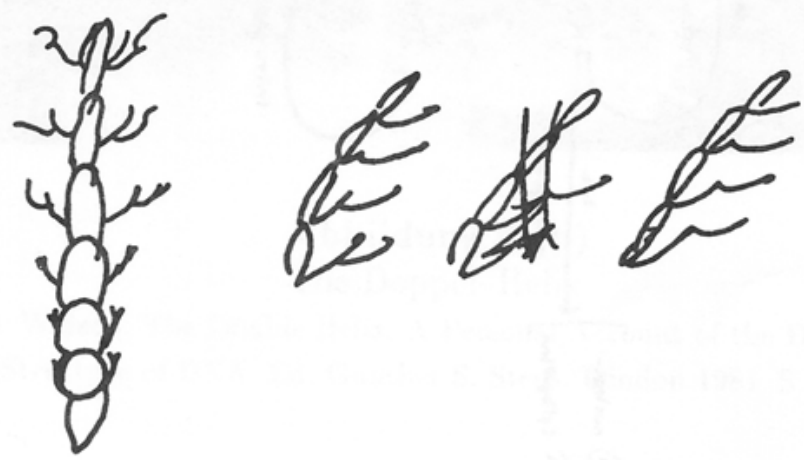

180 Hier trit nun die Lehre von den Verschiednen Häuten ein.

Abb. 2c: Skizze zur Metamorphose (aus: J.W. von Goethe, Die Schriften zur Naturwissenschaft (Leopoldina). Bd. 9A: Zur Morphologie. Bearb. von Dorothea Kuhn. Weimar 1977: 59) 


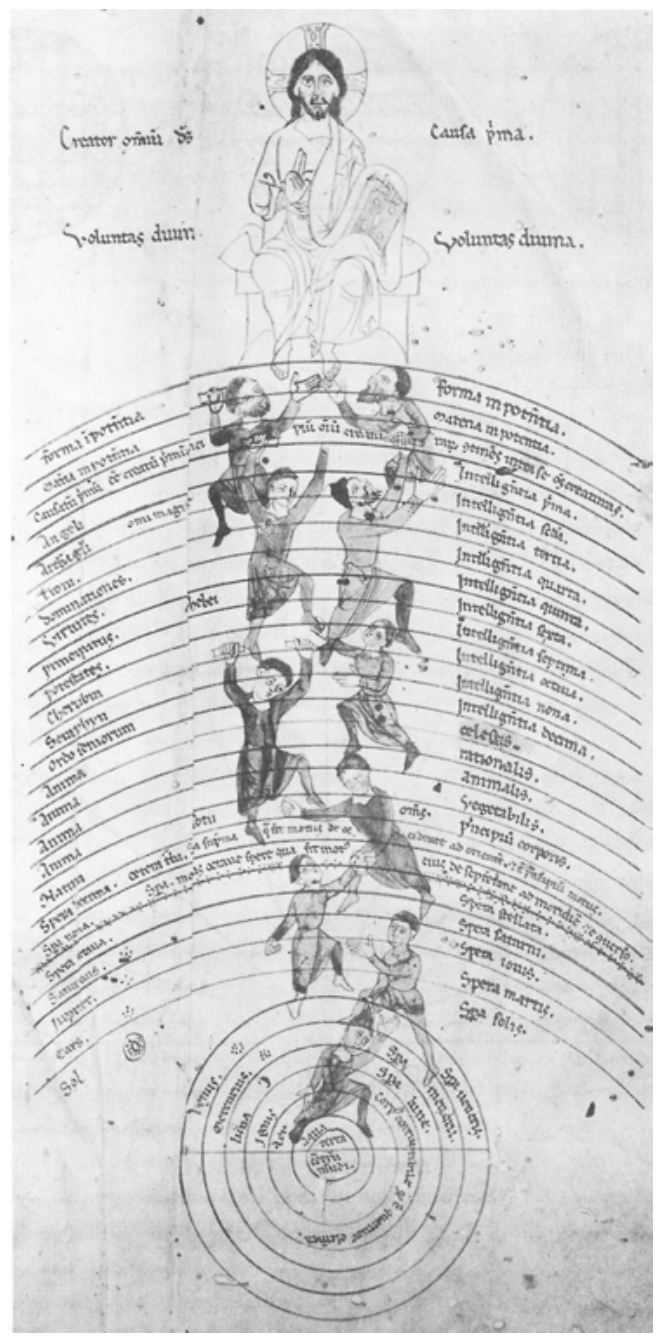

Abb. 3: Aufstieg der Seele durch die Sphären, Hermetisches MS., Anonymus, 12. Jahrhundert (aus: J. Purce, Die Spirale - Symbol der Seelenreise. München 1988, Abb. 36)

In der hermetischen Version [...] schraubt sich die Seele empor zu Gott, der über der Welt thront. In der Umkehrung des ursprünglichen Schöpfungsvorgangs durchquert der Mensch alle Zwischensphären, die Stufen allmählicher Erleuchtung. Von der Erde aus wandert er durch die vier Elemente, die Planetenbahnen, die Fixsterne, die Bewegungsprinzipien, die Pflanzen-, Tier-, Vernunft- und Himmelseele, die zehn Verstandes- oder Erkenntnisfähigkeiten und die ihnen entsprechenden Engelsreiche, das ersterschaffene Wesen oder den Weltgeist, die reine potentielle Materie und Form und schließlich zu Gott, der ersten Ursache und dem Schöpfer aller Dinge. 

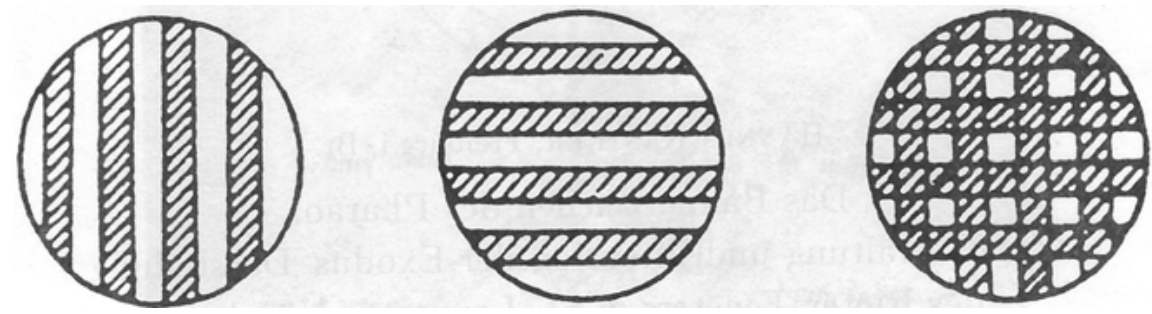

Abb. 4a: Sphärenabdeckung nach Bühler (aus: K. Bühler, Sprachtheorie. 2. Aufl. Stuttgart 1965: 348)

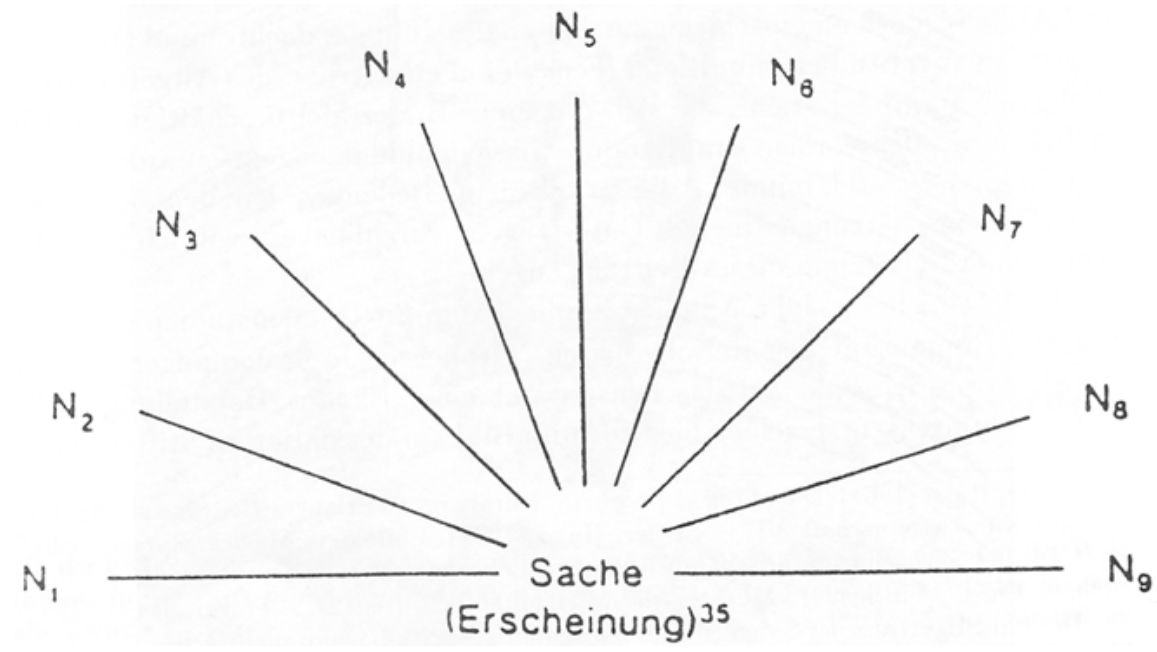

Abb. 4b: Namenbündelung nach Hartmann (aus: P. Hartmann, Das Wort als Name. Köln/Opladen 1958: 52) 